\title{
PERFORMANCE ENHANCEMENT OF SCM-OFDM SYSTEM USING CARRIER FREQUENCY OFFSET ESTIMATION AND CORRECTION
}

\author{
Rashmi $\mathrm{N}^{1}$ and Mrinal Sarvagya ${ }^{2}$ \\ ${ }^{1}$ Department of Electronics and Communication Engineering, \\ BMS Institute of Technology and Management ${ }^{1}$, India \\ ${ }^{2}$ Department of Electronics and Communication Engineering, \\ Reva university India \\ ${ }^{1}$ rashmiswamy@bmsit.in, ${ }^{2}$ mrinalsarvagya@ reva.edu.in
}

\begin{abstract}
OFDM system loses the orthogonality between subcarriers due to carrier frequency offset resulting in inter-carrier interference (ICI) in the OFDM system. CFO impedes the performance of the demodulation process in baseband receiver increasing BER in the OFDM system. In the proposed scheme, the estimation of CFO is performed using the Extended Kalman filter (EKF) and sigma pointed Kalman filter (SPKF) on the SCM-OFDM system. The simulation results show that the estimation using SPKF can achieve better results than other variants of the Kalman filter. The proposed system improves the BER performance of the OFDM system. Further, it is shown that SPKF outperforms than of EKF.
\end{abstract}

Keywords - Carrier Frequency Offset, OFDM, Superposition Coded Modulation, Sigma pointed Kalman filter

\section{INTRODUCTION}

The Orthogonal Frequency Division Multiplexing (OFDM) is a multicarrier bandwidth-efficient system, a promising technique for the systems which aspire for high rate transmission such as 4G-LTE advanced, Wimax, etc, Advantages of OFDM attracted several researchers and academicians to choose OFDM technique which can cater for high data rate applications. OFDM is also robust to multipath fading channels with low complexity implementation. OFDM uses higher modulation schemes such as PSK or QAM to achieve bandwidth efficiency. However, the Quadrature part of the PSK and QAM are not Gaussian distributed. To achieve channel capacity, the input to the channel must be Gaussian. Traditionally active signal shaping is performed on modulation schemes to achieve Gaussian distribution. Several methods were proposed by researchers and academicians to increase channel capacity [1]. With active signal shaping techniques the complexity at the transmitter and receiver increases. With APP decoder for bijective modulation (one to one mapping) schemes, the complexity increases with an increasing number of information bits as $\mathrm{O}\left(2^{\mathrm{N}}\right)$. In 1997, Daunt et al. designed a multilevel coded modulation scheme in [2-3] and pointed out that huge signal constellation and active signal shaping are indeed not mandatory to approach the channel capacity. Li Ping followed this work and did extensive analysis in [4]. Superposition coded modulation with one layer can achieve similar performance as presented by the authors of [4]. But the results are limited to 2 bps per signal dimension. Superposition Coded Modulation (SCM) is emerging as non-bijective, bandwidth and power efficient coded modulation with

Received:September 18, 2019

Reviewed: December 2, 2019

Accepted: December 10, 2019

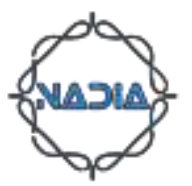


Gaussian quadrature components. The shaping gain in SCM is inherent without any additional complexity at the transmitter side. At the receiver side, the complexity is low as $\mathrm{O}(\mathrm{N})$ when equal power is allocated to the symbols. The hybrid combination of multilayer SCM and OFDM can achieve greater data rates compared to a single carrier OFDM system. However, the challenge in OFDM is to maintain the orthogonality of the subcarrier. OFDM is highly prone to the timing errors and Carrier Frequency Offsets (CFO) which is caused mainly due to 1) mismatch between the transmitter and local oscillator frequencies at the receiver 2) Phase rotation caused due to Doppler Effect. The inaccurate CFO estimation destroys the orthogonality among subcarriers leads to InterCarrier Interference (ICI). Doppler effect is significant in an aeronautical communications application, for example, CFO is high as 0.54 , at a $2.5 \mathrm{GHz}$ as carrier frequency, vehicle speed $120 \mathrm{KM} / \mathrm{Hr}$ with 1024 subcarriers OFDM system. Hence apposite methods are imperative for the successful utilization of OFDM in such a highspeed environment. In this connection, several methods were proposed in literature such as the Successive Interference Cancellation (SIC) method proposed by [7]. In this method, at the transmitter for adjacent subcarriers are assigned with opposite symbols which are subtracted at the receiver, advanced SIC method, in which phase rotation is done before sending is proposed in [8]. To mitigate the ICI, Carrier Frequency Offset(CFO) is necessary, CFO estimation using the Maximum Likelihood estimator is proposed in[9], which increases the complexity of the receiver with an increase in the order of modulation. Several Kalman filter approaches are proposed in [10] to estimate CFO the results obtained were significant. Since CFO is nonlinear, linear approaches result in low performance of the OFDM system. EM algorithm proposed in [11] for estimation of CFO, exhibit computational complexity at the receiver, due to computationally intense detection at the receiver.

In [12] Particle filter was proposed, however, the particle approaches have occasionally particle insolvency (PI) problem. Due to the re-sampling process particle insolvency problem arises. In a dynamic system, if the state process noise is small, the PI problem increases. However, we can avoid the PI problem by modeling state process noise as zero in $\mathrm{CFO}$ estimation.

To estimate the CFO, the Extended Kalman filter is proposed in [13], but the computational complexity is more in the Extended Kalman filter, which results in the OFDM power inefficient receiver.

In this paper, the Unscented Kalman filter (UKF) also referred to as sigma point Kalman filter (SPKF) is proposed for investigating the performance of the system under consideration for the CFO problem [14-15]. UKF varies from CDKF in the calculation of sigma points and covariances.

The paper is structured as follows: the system model is presented in section II, we present a state-space model of CFO in SCM-OFDM in section III. In section IV we present a brief explanation of the Extended Kalman filter and outlines UKF using Sigma Points and section $\mathrm{V}$ the simulation results of the performance of the SCM-OFDM system with CFO estimation using UKF are presented and section VI is the conclusion.

\section{SYSTEM MODEL}

The serial data stream is divided into L- parallel data stream each layer is separately encoded, randomly interleaved and modulated with QPSK modulation. Quadrature phaseshift keying (QPSK) symbols.

$$
x_{l}^{L}=x_{r e, j}^{L}+i x_{i m, j}^{L}
$$


$x_{l}^{L}:$ QPSK symbol

The QPSK sequences are linearly superimposed to form $\mathrm{x}_{\mathrm{j}}$.

$$
\mathrm{X}_{\mathrm{j}}=\sum_{l=0}^{L-1} \rho_{l} e^{j \theta_{l} \mathbf{X}_{l}^{\mathrm{L}}}
$$

Where $\rho_{l}>0$ is an amplitude factor, $\theta_{l}\left(0 \leq \theta_{l} \leq \frac{\pi}{2}\right)$ for layer -1 .

The superimposed symbols are fed to the OFDM modulator point consists of N-point Discrete Fourier Transforms (IDFT) unit followed $\mathrm{N}_{\mathrm{g}}$-point Cyclic Prefix(CP) to evade Inter Symbol Interference(ISI)[16][17]. The IDFT

output is collected as vector $\mathrm{D}_{\mathrm{i}}=\left[\mathrm{D}_{\mathrm{i}}(0), \mathrm{D}_{\mathrm{i}}(1), \ldots \mathrm{D}_{\mathrm{i}}(\mathrm{N}-1)\right]^{\mathrm{T}}$. A cyclic prefix is appended to $D_{i}$ such that

$D_{i}(k)=D_{i}(k+N)$ for $-N_{g} \leq k \leq-1$.

$\mathrm{D}_{\mathrm{i}}(\mathrm{k})=\left\{\frac{1}{\sqrt{N}} \sum_{n=0}^{N-1} X_{i}(k) e^{j 2 \pi n k / N},\right\} ; \mathrm{k}=0,1, \ldots \mathrm{N}-1$

Where $X_{i}(k)$ are superimposed modulated data symbols assigned to $\mathrm{N}$ subcarriers and with $\mathrm{N}_{\mathrm{g}}$ as the length of $\mathrm{CP},-\mathrm{Ng}<\mathrm{n}<\mathrm{N}-1$.

The SCM-OFDM signal propagates through the multipath channel, the $\mathrm{L}$ - tap channel impulse response is given by $h_{l}(l=0,1, \ldots . L-1)^{\mathrm{T}}$.

The received signal can be articulated as:

$y_{\mathrm{k}}=e^{\frac{j 2 \pi \epsilon k}{N}}\left(\sum_{l=0}^{L-1} D_{k-l} h_{l}\right)+w_{k}$

$w_{k}$ is the AWGN

' $\epsilon$ ' is a normalized carrier frequency offset. Where ' $\varepsilon^{\prime}$ needs to be estimated, to restore the orthogonality in the OFDM symbol. The CFO calculated with $f_{d}$ as the Doppler frequency expressed as

$f_{d}=\frac{C V_{r} F_{C}}{C}$

Where $v_{r}$-the speed of the mobile station, C- the speed of light,

$\mathrm{f}_{\mathrm{c}^{-}}$carrier frequency

$\epsilon=f_{d} / \Delta f=N f_{d} / f_{s}$

$\mathrm{f}_{\mathrm{s}}$ is the subcarrier spacing of $D_{i}(k)$.

\section{DYNAMIC STATE SPACE MODEL OF CFO}

In slow time-varying channel carrier frequency offset is considered as constant for OFDM symbol duration.

The state and measurement equations for dynamic state system are as follows:

$$
\begin{array}{r}
a_{k}=s_{k}\left[a_{k-1}\right]+u_{k,} \\
y_{k}=f_{k}\left[a_{k}\right]+w_{k}
\end{array}
$$


Where $f_{k}($.$) is the observation function a, s_{k}($.$) is the state transition function, u_{k}$ and $w_{k}$ is the process noise and measurement noise respectively. Corresponding state and measurement equations are:

$$
\begin{aligned}
& \epsilon_{k}=\epsilon_{k-1} \\
& \mathrm{y}_{\mathrm{k}}=\mathrm{f}_{\mathrm{k}}(\varepsilon)+\mathrm{w}_{\mathrm{k}} \\
& \quad \mathrm{f}_{\mathrm{k}}(\varepsilon)=\mathrm{e}^{\frac{\mathrm{j} 2 \pi \mathrm{k} \epsilon}{\mathrm{N}}} \sum_{\mathrm{l}=0}^{\mathrm{L}-1} \mathrm{a}_{\mathrm{k}-1} \mathrm{~h}_{\mathrm{l}}
\end{aligned}
$$

Where $\varepsilon$ the normalized CFO and $\mathrm{w}_{\mathrm{k}}$ is additive noise. UKF estimates $\mathrm{a}_{\mathrm{k}}$ in succession from the observed measurement $y_{k}$.

Another popular Kalman filter EKF propagates the Gaussian Random Variable (GRV) with the first-order linearization of non-linear equation (11) through the system dynamics [18-19]. The evaluation of mean and covariance matrices of GRV using EKF leads to errors when the first-order approximation used. In UKF Gaussian distribution is approximated with state distribution by a set of sigma points. Hence we propose the UKF for better convergence and higher accuracy in CFO estimation.

\section{A) EXTENDED KALMAN FILTER}

The Kalman filter is a recursive estimator, computes the estimate of the current state based on the knowledge of previously estimated state and current measurement.

The dynamic state model for the CFO in vector form is represented as follow:

$$
\varepsilon(n)=\varepsilon(n-1)+u(n)
$$

' $\varepsilon$ ' is the normalized CFO and $\mathrm{u}(\mathrm{n})$ is a Gaussian process with mean equals to zero and variance equals to $\sigma^{2}$. The extended Kalman filter minimizes and measurement noises.

The EKF equations are as follows:

Time update:

$$
\begin{aligned}
& \epsilon(\mathrm{n})=\epsilon(\mathrm{n}-1)+\mathrm{u}(\mathrm{n}) \\
& \widehat{\epsilon}(\mathrm{n} \mid \mathrm{n}-1)=\widehat{\epsilon}(\mathrm{n}-1 \mid \mathrm{n}-1) \\
& \mathrm{P}(\mathrm{n} \mid \mathrm{n}-1)=\mathrm{P}(\mathrm{n}-1 \mid \mathrm{n}-1)+\sigma^{2}
\end{aligned}
$$

Measurement update:

$$
\begin{gathered}
\widehat{\epsilon}(\mathrm{n} \mid \mathrm{n})=\widehat{\epsilon}(\mathrm{n} \mid \mathrm{n}-1)+\mathrm{K}(\mathrm{n})(\mathrm{R}(\mathrm{n})-(\widehat{\epsilon}(\mathrm{n} \mid \mathrm{n}-1)) \\
\mathrm{P}(\mathrm{n} \mid \mathrm{n})=[1-\mathrm{K}(\mathrm{n})] \mathrm{P}(\mathrm{n} \mid \mathrm{n}-1)
\end{gathered}
$$

Kalman gain is given by

$$
\mathrm{K}(\mathrm{n})=\mathrm{P}(\mathrm{n})[\mathrm{P}(\mathrm{n})+1]^{-1}
$$

Where ' $\mathrm{K}$ ' is Kalman gain, $\mathrm{P}(\mathrm{n} \mid \mathrm{n}-1)$ and $\mathrm{P}(\mathrm{n} \mid \mathrm{n})$ prior and posterior MSE ' $\epsilon$ ' normalized $\mathrm{CFO}$ to be estimated.

\section{B) SIGMA POINTED KALMAN FILTER:}

The gain and error variance from (9) and (10) are related as follows: 
Let $\rho=1$ be the length of the state vector.

$$
\begin{aligned}
& \mathrm{P}_{\mathrm{i}}(\mathrm{n}+1 \mid \mathrm{n})=\mathrm{P}_{\mathrm{i}}(\mathrm{n} \mid \mathrm{n})+\sigma_{w}^{2} \\
& \mathrm{P}_{\mathrm{i}}(\mathrm{n} \mid \mathrm{n})=\mathrm{P}_{\mathrm{i}}(\mathrm{n} \mid \mathrm{n}-1)-\mathrm{K}_{\mathrm{i}}(\mathrm{n}) P_{i}^{r}(n) K_{i}^{*}(\mathrm{n})
\end{aligned}
$$

Where

$$
K_{i}(n)=\left\{P_{i}^{e r}(n)\right\}\left\{P_{i}^{r}(n)\right\}^{-1}
$$

$K_{i}$ is the gain of the UKF filter, $P_{i}^{e r}(n)$ is the covariance between the state prediction error and innovation.

$$
\begin{aligned}
& \breve{r_{l}}(n)=r_{i}(n)-\hat{r}_{i}(\mathrm{n}), \\
& \quad \text { where } \hat{r}_{i}(\mathrm{n})=\hat{R}_{i}(n) \\
& X_{i}(n \mid n-1)=\left[\begin{array}{ll}
X_{0, i}(n \mid n-1) X_{1, i}(n \mid n-1) & X_{2, i}(n \mid n-1)
\end{array}\right] \\
& \quad=\left[\begin{array}{ll}
\hat{\epsilon}_{i}(n-1) & \hat{\epsilon}_{i}(n-1)+\mu \sqrt{P_{i}}(n-1) \hat{\epsilon}_{i}(n-1)-\mu \sqrt{P_{i}}(n-1)
\end{array}\right]
\end{aligned}
$$

Where $\mu=\sqrt{1+k}, k=\alpha^{2}-1$ and $10^{-3} \leq \alpha \leq 1$

$\hat{\epsilon}_{i}(n \mid n-1)=\sum_{n=0}^{2} w_{n} X_{n, i}(n \mid n-1)$

Where $w_{0}=k /(1-k)$ and $w_{n}=1 /[2(1+k)]$

The sigma points are propagated to nonlinear function eq. (11) and rewritten as follows

$\mathrm{Y}_{\mathrm{i}}(\mathrm{n} \mid \mathrm{n}-1)=f\left\{X_{i}(n \mid n-1)\right\}$

The measurement update in time:

$\hat{r}_{i}(\mathrm{n})=\sum_{n=0}^{2} w_{n} Y_{n, i}(n \mid n-1)$

The sigma points are promulgated to nonlinear function(eq(11))is rewritten as follows

$$
\mathrm{Y}_{\mathrm{i}}(\mathrm{n} \mid \mathrm{n}-1)=f\left\{X_{i}(n \mid n-1)\right\}
$$

The measurement update in time:

$$
\hat{r}_{i}(\mathrm{n})=\sum_{n=0}^{2} w_{n} Y_{n, i}(n \mid n-1)
$$

$\hat{\epsilon}_{i}(n \mid n)=\hat{\epsilon}_{i}(n \mid n-1)+\operatorname{Re}\left(K_{i}(n)\left\{r_{i}(n)-\hat{r}_{i}(n)\right\}\right)$

\section{SIMULATION}

The SCM-OFDM system is simulated with 12 subcarriers,

$\mathrm{N}_{\mathrm{g}}=128$ (the number of a cyclic prefix). Carrier frequency $\mathrm{f}_{\mathrm{c}}=2.5 \mathrm{GHz}$ and channel bandwidth $\mathrm{B}=10 \mathrm{MHz}$. For simulation normalized CFO due to Doppler Effect and user movement is considered. For simulation Rayleigh channel is considered, BPSK is used to modulate the information bits in each of the SCM layers. We assume the CFO and channel estimation have been performed on the preamble. The sigma pointed Kalman filter aims at estimating the CFOs for the SCM-OFDM symbol. We carried out the testing of the SCM-OFDM system for estimating CFO with SPKF and EKF.

The results showed in figure 3, Sigma pointed Kalman filter is tracking the CFO better than the Extended Kalman filter. According to figure 1. Sigma pointed Kalman filter is accurately estimating the CFO than the Extended Kalman filter. 
The percentage of error in estimating CFO using EKF is $11.01 \%$ whereas using SPKF is $0.24 \%$. There is a significant reduction in error. Hence proposed novel scheme proves to be a powerful scheme to increase throughput by reducing BER.

The CFO effect on OFDM and SCM-OFDM without Kalman filters estimation is shown in figure 2. The result shows that the performance of the OFDM system is degraded. At the SNR 5dB using SPKF $4.76 \%$ of BER reduction is achieved on the SCM-OFDM scheme. Hence data rate is also increased by an amount of $1.14 \%$ compared to using EKF on the SCM-OFDM system.

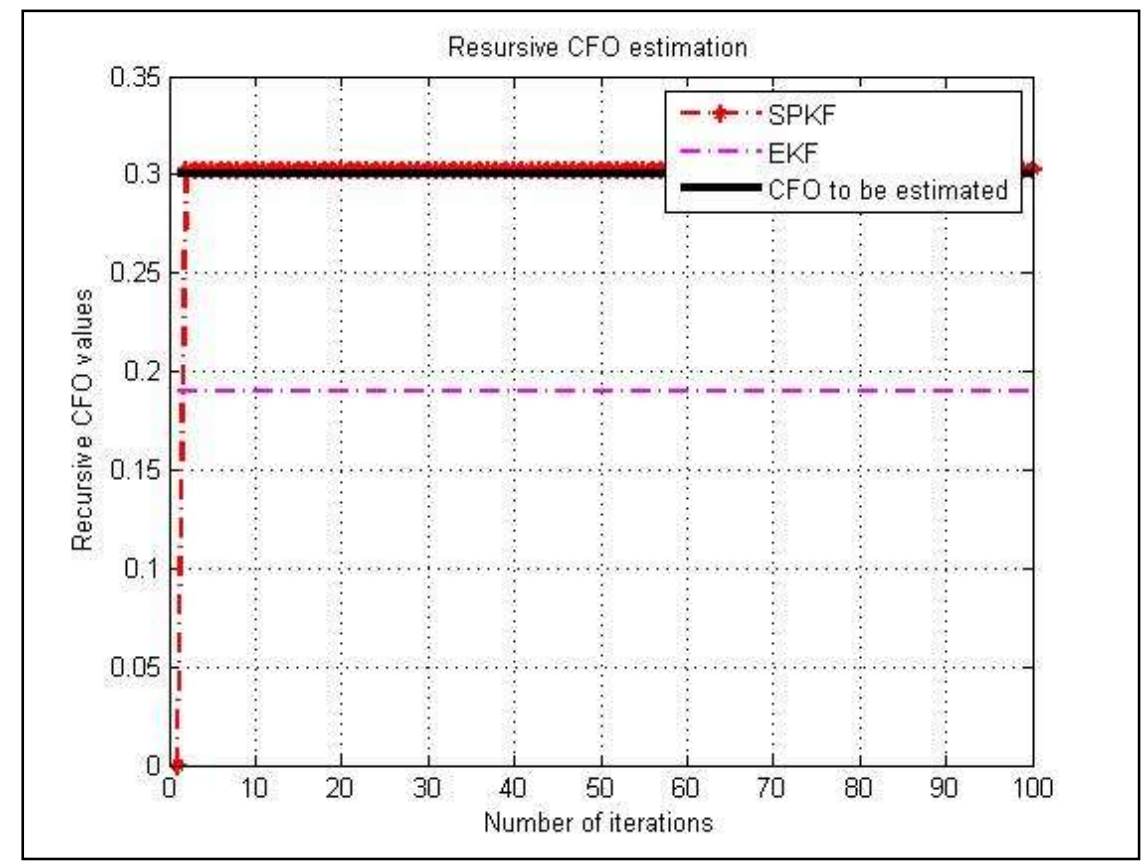

Fig. 1 Comparison of Sigma point Kalman Filter and Extended Kalman Filter for CFO estimation over normalized $\mathrm{CFO}=0.3$ in the SCM-OFDM system.

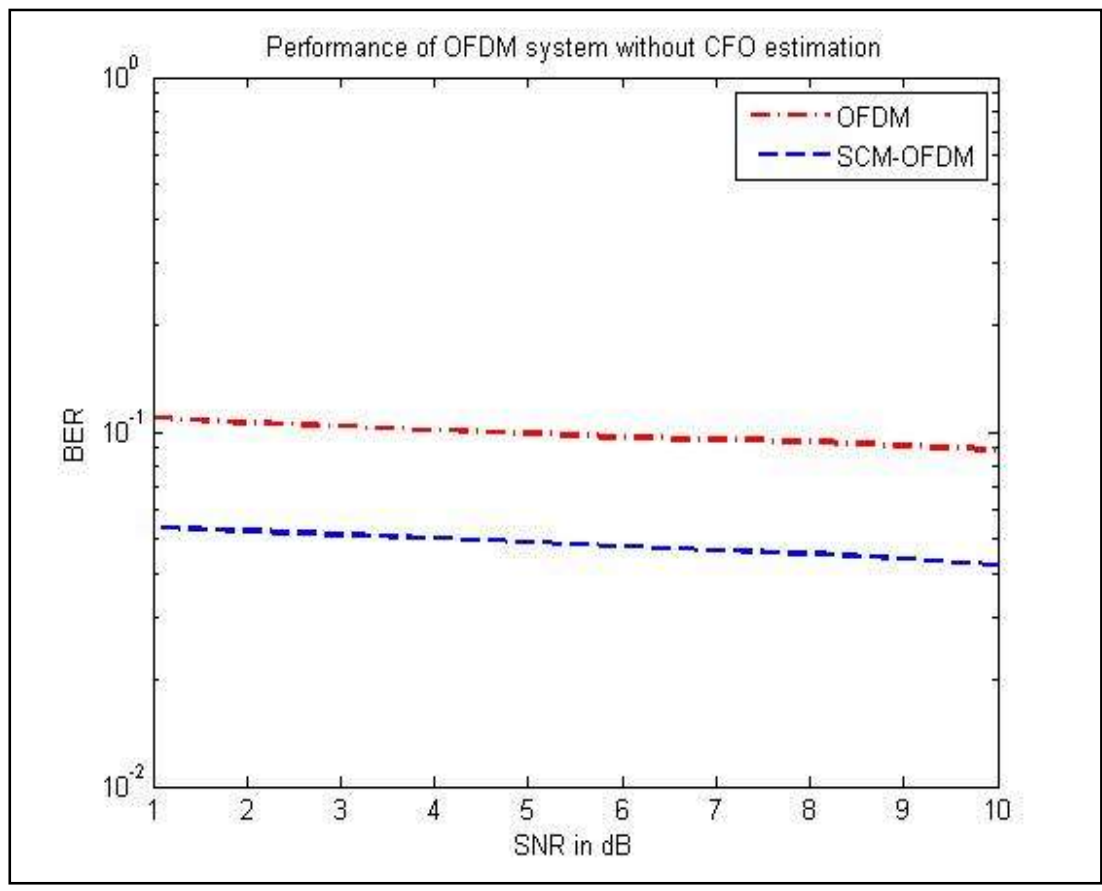

Fig. 2 Performance of OFDM and SCM-OFDM system without CFO estimation 
Table I. Error variance for EKF and SPKF with SCM-OFDM for CFO estimation

\begin{tabular}{|c|c|c|c|c|}
\hline $\begin{array}{l}\mathrm{E}_{\mathrm{b}} / \mathrm{N}_{\mathrm{o}} \\
(\mathrm{dB})\end{array}$ & $\begin{array}{l}\text { Theoretical } \\
\text { BER }\end{array}$ & $\begin{array}{l}\text { BER } \\
\text { (EKF) }\end{array}$ & $\begin{array}{l}\text { BER } \\
\text { (SPKF) }\end{array}$ & $\begin{array}{l}\% \\
\mathrm{~A}\end{array}$ \\
\hline 0 & $1.464 \times 10^{-1}$ & $0.769 \times 10^{-}$ & $\begin{array}{c}1.05628 \mathrm{X} \\
10^{-1}\end{array}$ & 40 \\
\hline 5 & $6.418 \times 10^{-2}$ & $6.96 \times 10^{-2}$ & $6.23 \times 10^{-2}$ & 18.8 \\
\hline 10 & $2.327 \times 10^{-2}$ & $5.49 \times 10^{-2}$ & $4.3 \times 10^{-2}$ & 200 \\
\hline
\end{tabular}

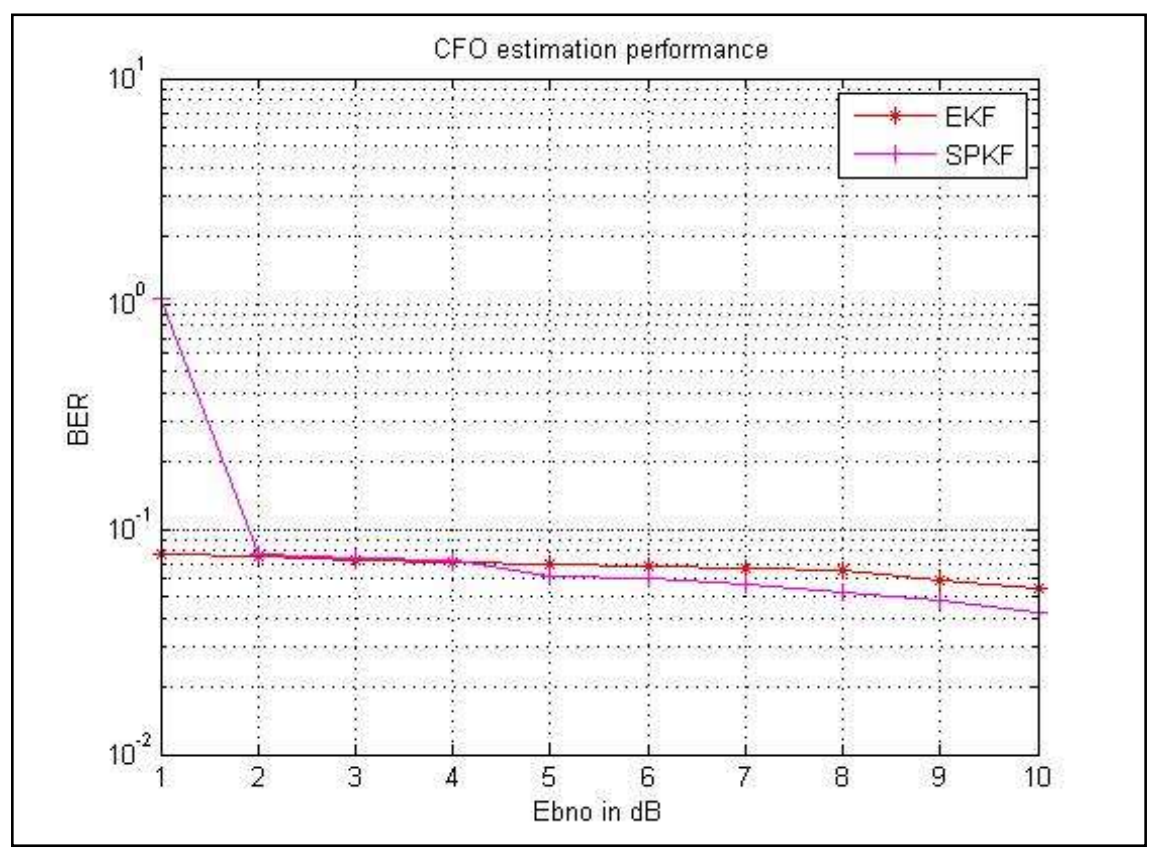

Fig. 3 BER performance using SPKF and EKF in the SCM-OFDM system

Figure 3 represents the SNR Vs BER performance of EKF and SPKF for normalized CFO estimation and correction for the SCM-OFDM system. The SPKF outperforms the EKF in BER reduction and thereby increasing the data rate and throughput by $1.14 \%$ compared to using EKF for estimating CFO with SCM-OFDM system

Table I presents the error variance of EKF and UKF, column $\mathbf{A}$ refers to the difference between theoretical BER and BER using SPKF and is defined as follows:

$$
M S E=\frac{1}{N} \sum_{i=1}^{N}\left(s_{i}-\hat{s}\right)^{2}
$$

$\mathrm{S}_{\mathrm{i}}$ refers to the estimation at each step and $\hat{s}$ is the true value. From Table I it is evident that sigma point Kalman filter performance is superior with the smallest estimation error.

\section{CONCLUSION}

The Superposition Coded Modulation -OFDM system is simulated with sigma pointed Kalman filter for estimating normalized CFO the results show that compared to the Extended Kalman filter, the performance of SPKF improved under time-varying channel with Doppler Effect. SPKF is suitable for the nonlinear environment. There is an improvement in data rate due to SPKF compare to EKF. The proposed scheme estimates 
the CFO nonlinearities and corrects the same. CFO estimation for SCM-OFDM system is imperative for the next generation communication technologies. As the next generation technology demands high data rates with a better quality of service.

Future scope: The performance of the SCM-OFDM system will be compared with the MIMO-OFDM system.

\section{REFERENCES}

[1] Liu, L., Liang, C., Ma, J., \& Ping, L. (2019). Capacity optimality of AMP in coded systems. arXiv preprint arXiv:1901.09559.

[2] Damrath, M., Hoeher, P. A., \& Forkel, G. J. (2018). Piecewise linear detection for direct superposition modulation. Digital Communications and Networks, 4(2), 98-105.

[3] Osamura, A., Song, G., Cheng, J., \& Cai, K. (2018, October). Sparse Multiple Access and Code Design with Near Channel Capacity Performance. In 2018 International Symposium on Information Theory and Its Applications (ISITA) (pp. 139-143). IEEE.

[4] Li, S., Bai, B., Zhou, J., He, Q., \& Li, Q. (2018). Superposition coded modulation based faster-thanNyquist signaling. Wireless Communications and Mobile Computing, 2018.

[5] Chi, Y., Liu, L., Guo, J., Song, G., Yuen, C., \& Guan, Y. L. (2019). Variable-Rate Coding With Constant BER for NOMA via Multilevel IRA Coding. IEEE Transactions on Vehicular Technology, 68(5), 5149-5153.

[6] Cho, H., Nguyen, T. H., Nguyen, H. N., Choi, H. K., Choi, J. K., \& Ro, S. (2018). A robust ICI suppression based on an adaptive equalizer for very fast time-varying channels in LTE-R systems. EURASIP Journal on Wireless Communications and Networking, 2018(1), 17.

[7] Vahidi, V., \& Saberinia, E. (2018). ICI mitigation for high-speed OFDM communications in highmobility vehicular channels. In Information Technology-New Generations (pp. 17-22). Springer, Cham.

[8] Lin, B., Tang, X., Ghassemlooy, Z., Li, Y., Zhang, M., Wu, Y., \& Li, H. (2019). A NOMA scheme for visible light communications with single carrier transmission and frequency-domain successive interference cancellation. Optik, 183, 445-450.

[9] Wang, Y. Y., Huang, C. W., \& Chen, W. W. (2018). Maximum likelihood carrier frequency offset estimation algorithm with adjustable frequency acquisition region. Journal of the Franklin Institute, 355(5), 2978-2985.

[10] Singh, S., Kumar, P., \& Prasad, S. V. A. V. (2019). Improvement of BER in Wavelet Transform-based OFDM System with Kalman Filter. Journal of Communication Engineering \& Systems, 4(1), 21-28.

[11] Zhang, X., Zhang, L., Xiao, P., \& Wei, J. (2019). Fine Timing Synchronization Based on Modified Expectation Maximization Clustering Algorithm for OFDM Systems. IEEE Wireless Communications Letters.

[12] Malarvezhi, P., \& Kumar, R. (2018). A Diversity Enhanced-Particle Filter based Multiple Carrier Frequency Offset Estimation Using Hadamard Matching in CA-OFDM Based LTE-A System. Wireless Personal Communications, 101(1), 87-99.

[13] Huu, N. N., Quoc, H. P., Van, L. P., Nguyen, T. H., Cho, H., Nguyen, V. D., \& Jeon, B. (2018, January). An OFDM System Design with Efficient ICI Cancellation over Embedded LTE-R Channels Based on the USRP Platform. In Proceedings of the 12th International Conference on Ubiquitous Information Management and Communication (p. 10). ACM.

[14] Liao, Y., Shen, X., Sun, G., Dai, X., \& Wan, S. (2019). EKF/UKF-based channel estimation for robust and reliable communications in V2V and IIoT. EURASIP Journal on Wireless Communications and Networking, 2019(1), 144.

[15] Shen, Z., Xu, K., Wang, Y., \& Xie, W. (2018, October). Angle-Domain Channel Tracking for HighSpeed Railway Communications with Massive ULA. In 2018 IEEE 18th International Conference on Communication Technology (ICCT) (pp. 159-165). IEEE.

[16] Rashmi, N., \& Sarvagya, M. (2016, March). Channel estimation using Extended Kalman filter for superposition coded modulation system. In 2016 3rd International Conference on Computing for Sustainable Global Development (INDIACom) (pp. 3482-3486). IEEE.

[17] Rashmi, N., \& Sarvagya, M. (2016). State Space Model-Based Channel Estimation using Extended Kalman Filter for Superposition Coded Modulation OFDM System. BVICA M's International Journal of Information Technology, 8(2), 1017.

[18] D J Costello and G D Forney "Channel coding: The Road to channel capacity," Proc. IEEE, vol.95, no.6, June 2007, pp.1150-77.

[19] L Duan, B. Rimoldi, and R. Urbanke, "Approaching the AWGN channel capacity without active shaping," in Proc. IEEE int. Symp.Information theory (ISIT'97), Ulm, Germany, Jun/Jul 1997, p.374. 